\title{
Biomarkers in individualized management of chimeric antigen receptor T cell therapy
}

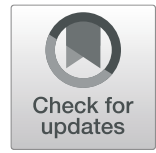

Mengyi Dư ${ }^{1}$ Parameswaran Hari ${ }^{2}, \mathrm{Yu} \mathrm{Hu}^{1}$ and Heng Mei ${ }^{*}$

\begin{abstract}
The development of chimeric antigen receptor (CAR) T cell immunotherapy has achieved promising results, both in clinical studies and in commercial products for patients with hematologic malignancies. Despite high remission rates of CAR-T cell therapy in previously untreatable, refractory and/or relapsed patients, several challenges in CAR-T therapy remain to be overcome, especially in integrating such therapies into personalized disease management approaches. Given the unique characteristics of CAR-T therapy, it is particularly urgent to identify biomarkers to maximize their clinical benefits. This systematic review summarizes clinically relevant biomarkers that may help individualized disease management in patients receiving CAR-T cell therapy in terms of toxicity warning, efficacy prediction and relapse monitoring. We summarize data from 18 clinical trials, including traditional indicators like cytokines, biochemical proteins, tumor burden, as well as potential novel indicators such as CAR-T cell expansion and persistency. The establishment of a biomarker-based system aimed at individualized management is recommended to guide better clinical application of CAR-T products.
\end{abstract}

Keywords: CAR-T therapy, Biomarker, Safety, Efficacy, Prognosis

\section{Background}

With no restriction to major histocompatibility complex (MHC) [1], chimeric antigen receptor (CAR) - T cells have been a breakthrough in personalized cancer therapy, especially in hematological malignancies. Since the development of the first generation 1 of CAR-T cells $[2,3]$, their structure has been optimized. Currently, fourth generation CAR-T cells are available [4], which provide higher response rates and longer remission duration. The potent anti-tumor effects of CAR-T cells $[5,6]$ led to accelerated regulatory approval, an extensive investigation of their mechanisms, and the development in clinical investigation of several CAR-T cells targeting different tumor-associated antigens. To date, two antiCD19 CAR-T products have been approved by the Food and Drug Administration (FDA) for human use, which

\footnotetext{
* Correspondence: hmei@hust.edu.cn

${ }^{1}$ Institute of Hematology, Union Hospital, Tongji Medical College, Huazhong University of Science and Technology, Wuhan 430022, China

Full list of author information is available at the end of the article
}

are known as axicabtagene ciloleucel (axi-cel) and tisagenlecleucel (CTL019) [7]. After the first reports on CAR-T cells from the University of Pennsylvania, an increasing number of institutions around the world have reported the clinical trial of numerous CAR-T products, which are summarized in Table 1 . The anti-tumor effects of CD19 targeting CAR-T cells have been extensively explored and reported in patients with B-cell acute lymphocytic leukemia (B-ALL), chronic lymphocytic leukemia (CLL), non-Hodgkin's lymphoma (nonNHL), and other CD19 positive cancers [8]. The antitumor effects of CAR T-cells targeting the $\mathrm{B}$ cell maturation antigen (BCMA) have also been investigated in multiple myeloma (MM) [9]. The number of clinical trials involving the use of CAR-T registered on ClinicalTrials.gov (URL: https://clinicaltrials.gov/) is increasing exponentially. Except for CD19 and BCMA, various surface antigens, including CD22, CD20, and CD138, have also been purposed as therapeutic targets in lymphoid tumors [10-12], while CD123, CD33, CD56, and Fms- 


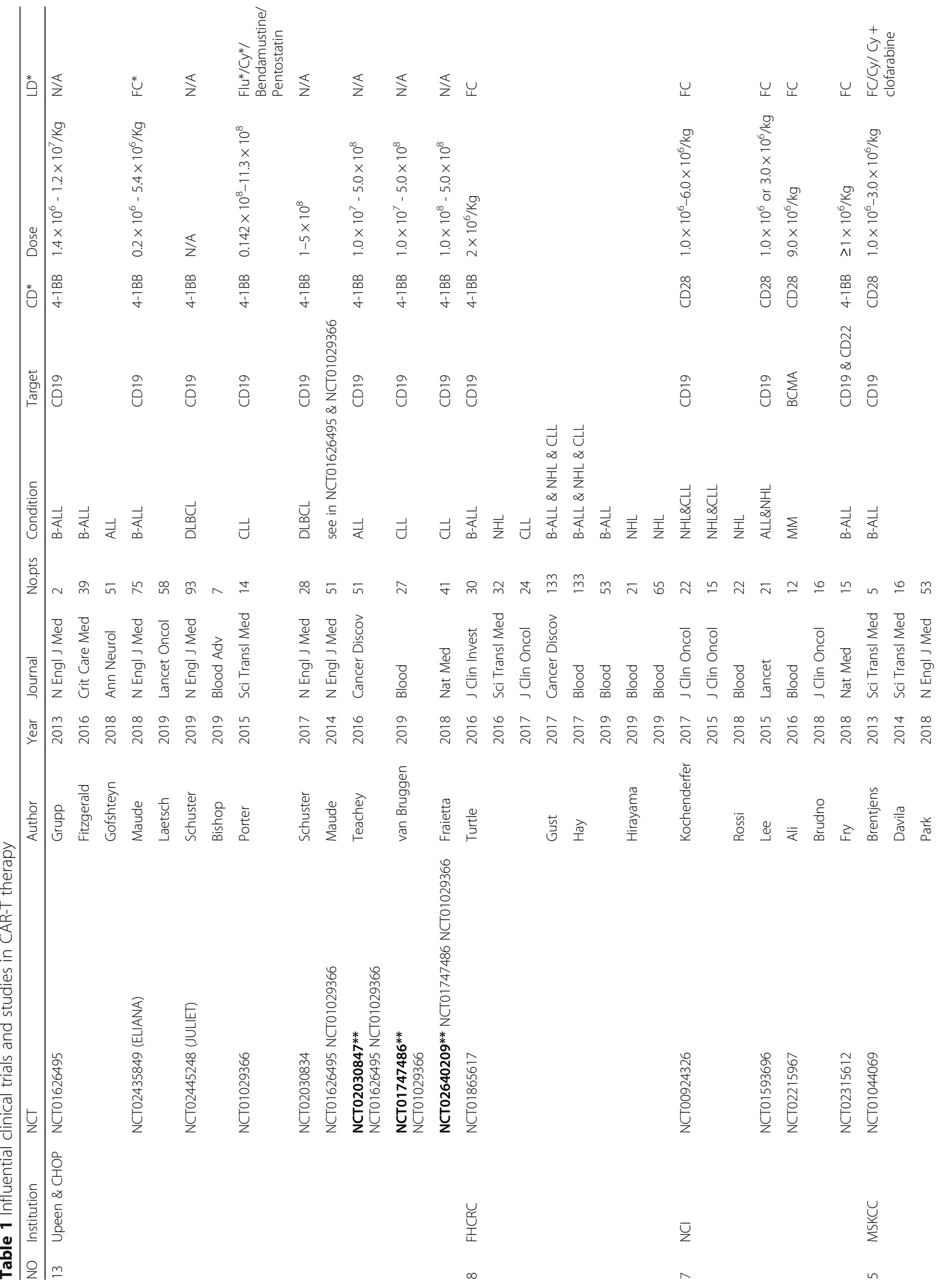




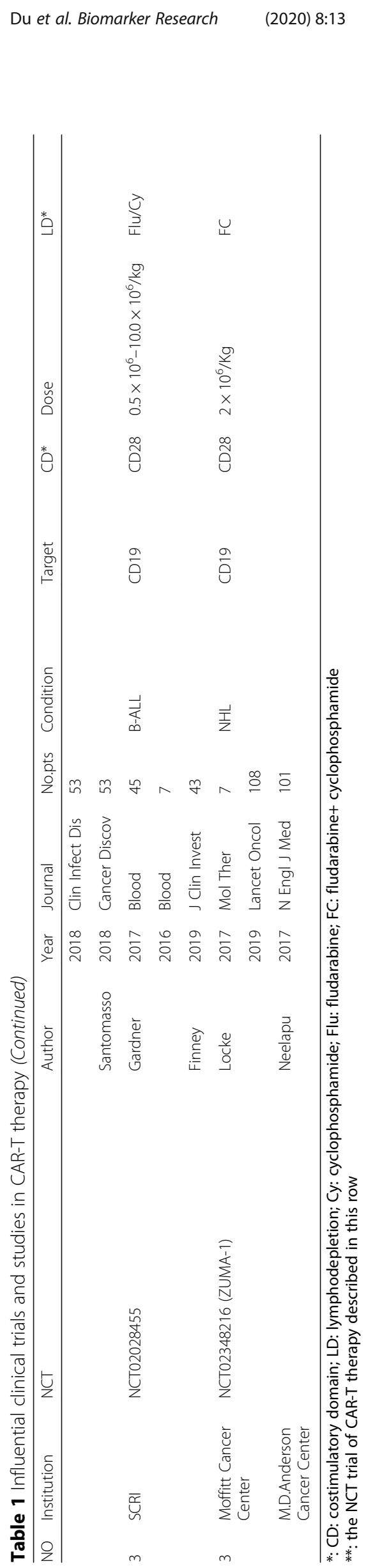

Page 3 of 13 
like tyrosine kinase (FLT3) have been suggested as targets in myeloid neoplasms $[13,14]$. The increasing commercial value of CAR T-cell therapies is also reflected in the fact that the number of patents on different CAR Tcell products has increased from less than 100 in 2013 to more than 600 in 2016 [15].

Severe cytokine release syndrome (CRS) and CARrelated encephalopathy syndrome (CRES) following CAR-T cell therapy can be life-threatening in some cases [16]. Moreover, it remains unclear to date why some patients exhibit impressive responses to CAR-T cells while others are resistant to such therapies [8]. Disease relapse following CAR-T cell therapy can occur in up to $50 \%$ of the patients by 12 months after infusion, and the mechanisms underlying the development of resistance to CAR$\mathrm{T}$ cells remain poorly understood [17]. The lack of robust predictive biomarkers of toxicity and efficacy are significant limiting the individualized management of patients undergoing treatment with CAR-T cells (Fig.1).

A biomarker is defined as "a characteristic that is objectively measured and evaluated as an indicator of normal biological processes, pathogenic processes, or pharmacological responses to therapeutic intervention" (From the National Institutes of Health Biomarkers Definitions Working Group 1998). In the context of CAR-T cell therapies, the "drug" varies between individuals and is capable of self-replication or expansion. Hence, the clinical benefit is heavily dependent on the characteristics of CAR-T cells themselves, including the quantity, function, and persistence prior to engineering, as well as their in vitro and post-infusion in vivo characteristics. These characteristics may serve as powerful predictors of the development of severe side effects, response rates, and duration of response or survival $[3,18,19]$, as they determine the properties $[20,21]$, intensity [22], and duration [23] of CAR-T cell effects. Furthermore, a variety of traditional indices have also been proposed as candidate biomarkers for CAR- $T$ cell therapies. These factors include inflammatory mediators of CRS and CRES [24, 25], markers of organ dysfunction due to severe toxicity [26], and factors associated with the primary disease [27]. In this review, we summarize and discuss various biomarkers that might have clinical value in predicting treatment outcomes and progression-free

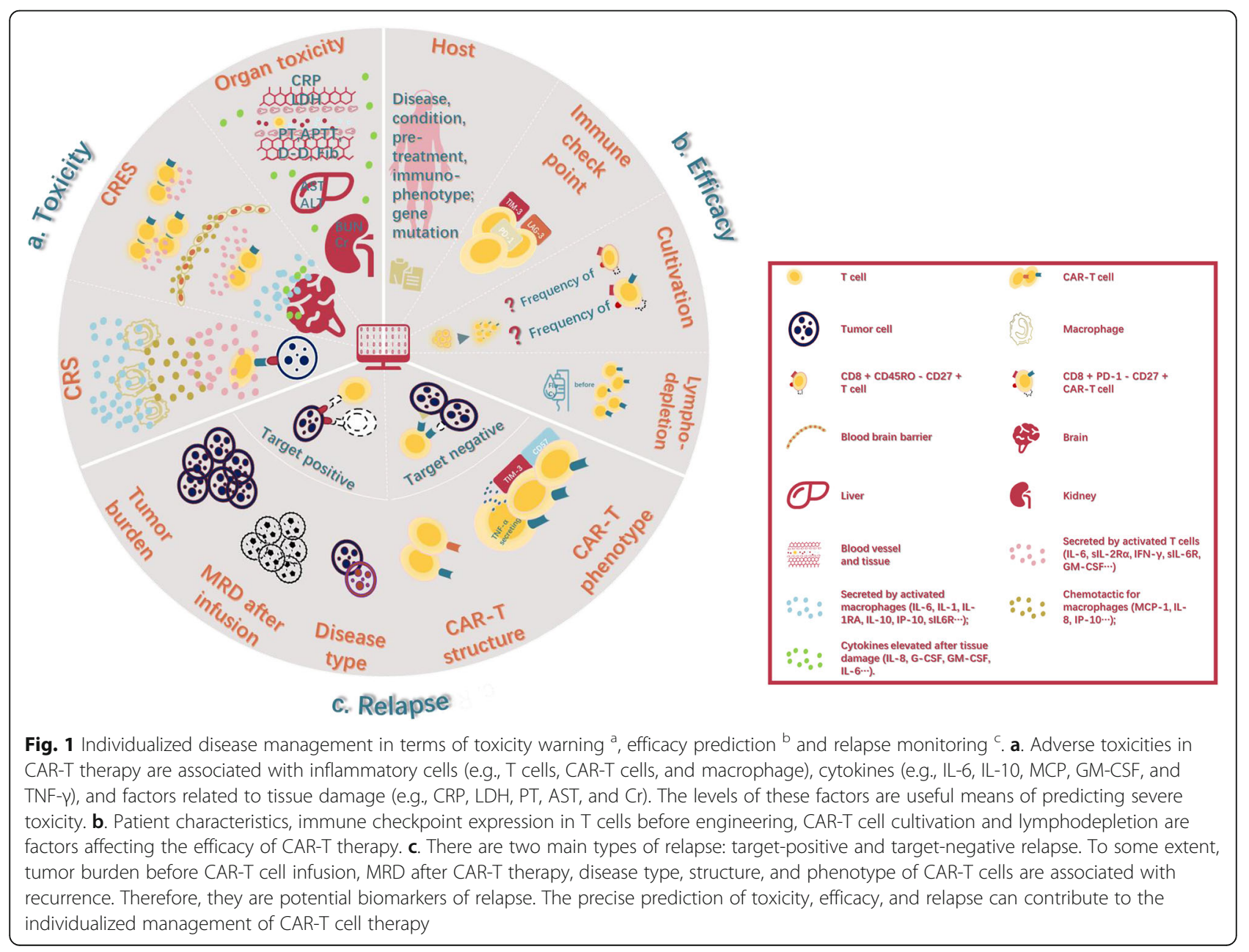


duration in patients treated with CAR-T cell therapies, thus contributing to the establishment of individualized patient management guidelines.

\section{Biomarkers predicting toxicity}

Safety is the primary endpoint in the majority of phase 1 clinical trials. Based on evidence from several studies [22, 24, 26, 28], CRS and neurotoxicity (CRES) have been suggested to be the most common side effects in patients treated with CAR-T cells. Thus, it is critical to identify biomarkers predicting the development of CRS and CRES.

\section{Cytokine release syndrome (CRS)}

The most common toxicity associated with CAR-T cell therapy is CRS, which is a form of systemic inflammatory response characterized by non-infectious fever, hypotension, hypoxia, and/or multiorgan toxicity. CRS usually develops in the first or second week after CAR-T infusion [29]. However, the severity of CRS symptoms can vary significantly, ranging from low grade with mild symptoms to high grade with early-onset high fevers, hyperpyrexia [26], high incidence of infections [30], and neurotoxicity [26]. Moreover, severe CRS can be accompanied by excessive macrophage activation, coagulation dysfunction, tumor lysis syndrome, and life-threatening multiorgan dysfunction [22-24, 31]. In terms of management, mild to moderate CRS is usually self-limited and can be controlled with close observation, hydration, and supportive care. In contrast, severe CRS requires intensive medical management with tocilizumab alone or in combination with steroids [31]. The management of CRS depends heavily on the risk grade system [32, 33]. Although the grading system takes into account various criteria [34], it is mainly based on clinical signs and subjective symptoms, which is not ideal for grading CRS, as its manifestation can vary immensely.

CRS is triggered by the activation of $\mathrm{T}$ cells after engagement of their CARs with cognate antigens expressed on the surface of tumor cells [35]. The activated $\mathrm{T}$ cells release various cytokines and chemokines, including interleukin (IL)-6, interferon (IFN)- $\gamma$, granulocyte-macrophage colony-stimulating factor (GM-CSF), and soluble IL-2R $\alpha$ $[24,36]$. These cytokines activate monocytes, macrophages and other immune cells, which, in return, release inflammatory cytokines, such as IL-1, IL-6, IL-10, IL-1RA, IFN- $\gamma$, and interferon $\gamma$-induced protein (IP)-10 [22, 24, $37,38]$ promoting tissue damage and multiorgan dysfunction [24]. The interaction between immune cells, tumor cells, cytokines, and necrotic tissue accelerate the pathophysiology of CRS [16]. Hence, CRS is determined by a complex network that includes cells (tumor cells, activated $\mathrm{T}$ cells or CAR - $\mathrm{T}$ cells, monocytes, and macrophages), inflammatory mediators, and other mediators released by damaged tissues or organs.

Based on the pathologic mechanisms underlying CRS, several groups of indicators have been recommended to monitor CRS. Inflammatory indicators, including cytokines, ferritin, and C-reactive protein (CRP), are the most commonly used predictive biomarkers [39]. The expression profile of cytokines have been investigated in B-ALL [22, 24], CLL [40], and diffuse large B-cell lymphoma (DLBCL) patients [25] treated with anti-CD19 CAR-T cell therapy, in an effort to identify predictive factors for CRS [24]. Peak levels of 24 cytokines were increased in patients and associated with severe CRS, while other 19 cytokines were of no statistically difference, such as IL-2 [24]. The cytokines associated with severe CRS could be divided into four categories: $\mathrm{T}$ cell-activating cytokines (e.g., IL-6, IFN- $\gamma$, sIL-2R $\alpha$, sIL-6R, and GM-CSF), monocyte/macrophage-activating cytokines (e.g., IL-6, IL1Ra, IL-10, IP-10, and sIL-6R), monocyte/macrophageattracting chemokines (e.g., monocyte chemoattractant protein (MCP)-1, IP-10, and IL-8), and cytokines released after tissue damage (e.g., IL-6, IL-8, granulocyte ColonyStimulating Factor (G-CSF, and GM-CSF) [24, 40, 41]. Besides these cytokines, a positive association between the levels of CRP or ferritin and severe CRS was reported [23, 24, 40-42]. IL-6, CRP, ferritin, IL-10, IL-15, and MCP-1 have been investigated as predictive factors for CRS in large populations (Table 2). The predictive value of IFN- $\gamma$, IL-15, and GM-CSF has been inconclusive, likely due to differences in study design, patient populations, disease type, or the CAR-T cell platform used.

The cytokine IL- 6 is produced by a variety of cells and can promote $\mathrm{T}$ cell proliferation and $\mathrm{B}$ cell differentiation, as well as inhibit apoptosis and stimulate the production of the acute-phase proteins CRP and ferritin $[53,54]$. The levels of the chemotactic factors IL-8, MCP-1, and IP-10 may indicate mononuclear phagocyte activation in response to systemic inflammation and endothelial damage [24]. IFN- $\gamma$, G-CSF, and GM-CSF have been suggested as essential players in endothelial cell injury, which frequently occurs during inflammation-associated tissue damage [55]. On the other hand, IL-10 inhibits the ability of macrophages to stimulate cytokine secretion by $\mathrm{T}$ helper type 1 (Th1) cells [56], increasing the cytokine storm as a response. Two recent pre-clinical studies [37, 38 indicated that IL-6, IL-1, and macrophage-derived cytokines were critical factors determining CRS severity, while $\mathrm{T}$ cell-derived cytokines were less important. IL-1 might be a key player in the development of CRS, and it promotes the secretion of IL-6 and sIL-6R. These two studies also suggested that the cytokine profile associated with hemophagocytic lymph-histiocytosis/macrophage-activation syndrome (HLH/MAS) mirrors the cytokine profile observed in severe CRS [24] and that IL-2, which is 
Du et al. Biomarker Research

(2020) 8:13

Page 6 of 13

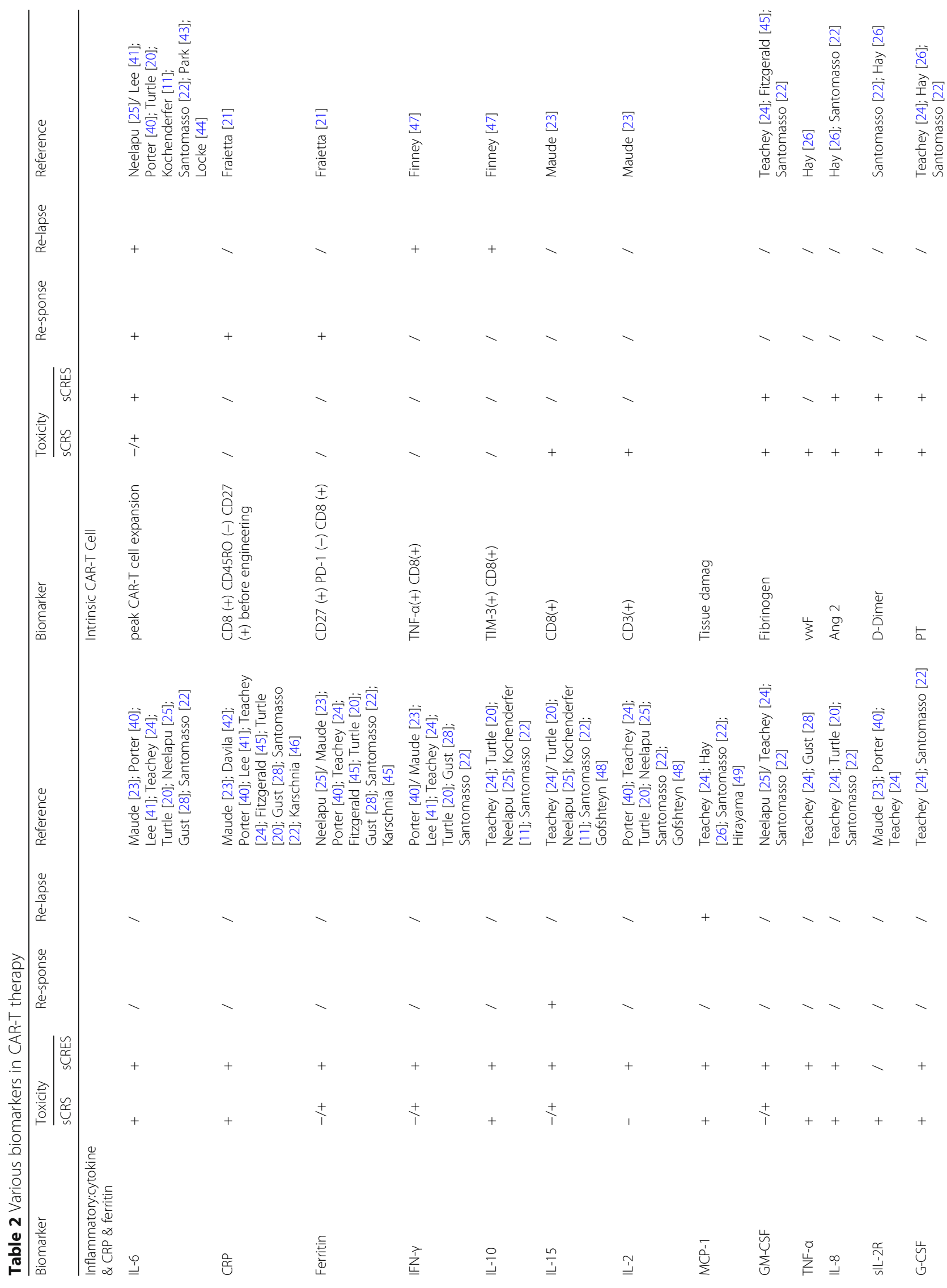




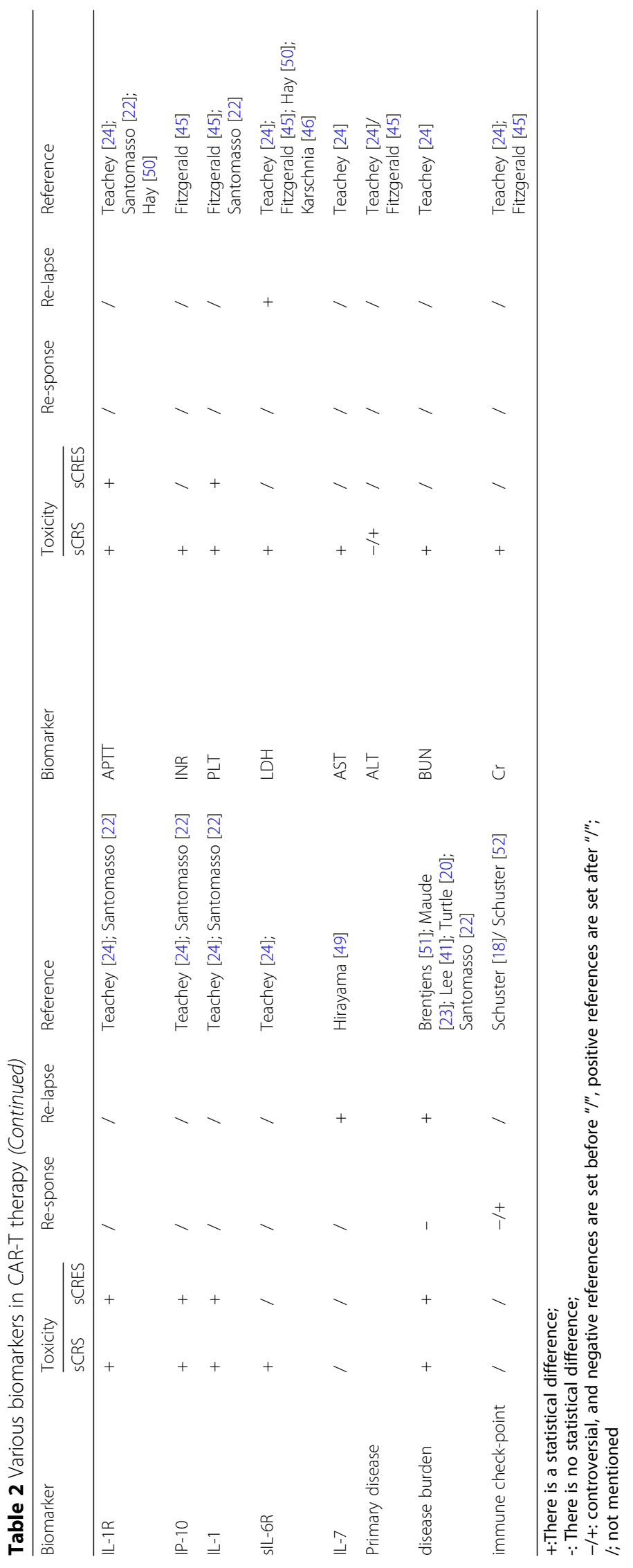


released only by $\mathrm{T}$ cells, does not associate with CRS severity [57].

Except for inflammatory factors, immune cells and tumor cells also play vital roles in the cytokine storm network. Notably, in biomarker discovery approaches, the fact that CAR-T are cell products rather than chemical compounds needs to be taken into consideration. CAR-T cells, upon binding to the appropriate antigen, they initiate a cascade of inflammatory reactions in vivo, hence intrinsic characteristic of CAR-T cells, such as the levels of CD3 and CD8 expression, could serve as potential biomarkers (Table 2) [28, 58-60]. The relationship between the peak levels of CAR T-cells and CRS severity remains controversial, likely emanating from the fact that macrophages and not activated $\mathrm{T}$ cells seem to be the key mediators of CRS [37, 38]. The predictive value of the characteristics of immune cells has been more extensively explored in efficacy than toxicity. Potent tumor cell elimination and high response rates may be coupled with a higher prevalence of severe CRS. As with tumor cells, disease burden is detected by cytology of bone marrow blasts or minimal residual disease (MRD). Interestingly, higher disease burden before enrollment [41] or at baseline [23] has been linked to a high risk of toxicity.

Coagulopathy, tumor lysis syndrome (TLS), HLH/ MAS, and organ dysfunction can develop during CRS [31], especially in patients with severe CRS. Except for HLH/MAS, which has similar pathology to CRS [24, 59], other symptoms or complications may develop due to tissue or organ damage, which correlates with the severity of CRS. Hay et al. demonstrated that the levels of angiopoietin-2 (Ang2) and von Willebrand factor (vwF) were increased in patients with severe CRS and that they could be used as predictive biomarkers. Ang2 and vwF are released from the WeibelPalade bodies upon endothelial activation, promoting capillary leak [61] and initiation of coagulation. Even though the mechanisms that lead to endothelial activation in CRS have not been fully characterized, the cytokines IL-6 and IFN- $\gamma$ seem to be involved [26]. Common indicators of abnormal coagulation, including prolonged prothrombin time (PT), activated partial thromboplastin time (APTT), elevated Ddimer, and hypofibrinogenemia, have been reported in grade $\geq 4$ CRS $[26,62]$. Lactate dehydrogenase (LDH) is a clinical biomarker of TLS $[63,64]$, and TLS is directly linked to disease burden [65]. This might explain why the peak levels of LDH associate with high-grade CRS [24] and why the median LDH level in grade 3 and 4 CRS patients associates with ferritin levels [45]. Besides, indicators of impaired liver and kidney function, such as AST and BUN, can be used as biomarkers of severe CRS [24, 45].

\section{CAR-related encephalopathy syndrome (CRES)}

CRES is another prominent toxicity occurring in 20$64 \%$ of patients undergoing CAR T-cell therapy, with a median onset of 4-5 days after CAR- $T$ cell infusion; in some cases, CRES can be concurrent with CRS [28]. The most common symptoms of CRES include encephalopathy, headache, delirium, anxiety, tremor, aphasia, and other neurotoxicity-related symptoms. Severe CRES (sCRES) emerges with earlier onset and is characterized by a longer duration and shorter progression-free survival (PFS) compared with mild CRES [46]. Though patients with high-grade CRS are more likely to develop high-grade CRES, fatal neurotoxicity may occur even in patients with mild CRS $[22,46]$. The treatment of CRES depends on the severity, which is defined according to the Common Terminology Criteria for Adverse Events (CTCAE) criteria [31, 34]. CRES management includes supportive care, or aggressive ICU-level care with antiepileptics, high-dose corticosteroids, and medications for status epilepticus and cerebral edema. Existing indicators alone are insufficient to predict CRES severity.

Thus far, the exact mechanisms of CAR-T cellassociated neurotoxicity remain poorly understood. Some studies have suggested direct toxicity by CAR-T cells and indirect toxicity caused by the increased levels of inflammatory cytokines in cerebrospinal fluid (CSF), which lead to endothelial cell activation and increased blood-brain barrier (BBB) permeability [28]. Several studies have suggested the presence of CAR-T cells in the central nervous system (CNS), yet it remains unclear why CAR-T cells migrate to the CNS in the absence of intracranial tumors $[23,41,42,66,67]$. Among all cytokines, IL- 1 and IL- 6 have been described as the key players of CRES [38]. More importantly, IL-1 precedes IL-6 secretion by many hours and is capable of inducing the secretion of IL-6 in circulating monocytes. Interestingly, anakinra, an IL-1 receptor antagonist, provided higher clinical benefit than tocilizumab in a murine model [38]. Based on currently available evidence, the pathological processes of CRES are mediated by inflammatory factors, CAR-T cells, and endothelial cells.

Several similarities between CRS and neurotoxicity have been described, including high cytokine concentrations in the serum, high CAR-T cell counts in the blood, and dysfunction of tissues and organs. Similarly to CRS, higher levels of inflammatory mediators, including IL-1, IL-6, IL-10, IL-2, IFN- $\gamma$, tumor necrosis factor (TNF)- $\alpha$, GM-CSF, G-CSF, MCP-1, CRP, and ferritin, are associate with higher grade neurotoxicity $[22,25,28,37,38$, $40,46,68]$. Santomasso et at. have indicated shown that patients with severe neurotoxicity had upregulated higher levels of IL- 1 and IL- 6 by day three after CAR-T cell infusion, suggesting that increased levels of these cytokines early after treatment can predict the early rise 
and higher peak of them were associated with severe CRES [22]. Despite the potent effects of IL-1 shown in pre-clinical studies [37, 38], seldom clinical studies have revealed its role in neurotoxicity in detail. Therefore, the role of IL-1 in immune responses and its potential use as a target to treat human diseases merits further investigation. The peak levels of MCP-1, IP-10, and IL-8 in CSF were more distinct higher than in the serum when compared with baseline level [22]. Elevated concentrations of MCP-1, IP-10, and IL-8 might be indicative of activated microglia, macrophages, or astrocytes in response to systemic inflammation and endothelial damage. Earlier elevation and higher peak levels of CAR-T cells in the blood have been associated with severe CRES $[22,28]$. Among the factors associated with CAR-T cell expansion, such as disease burden prior to infusion, the phenotype of CAR-T cells before and after genetic engineering, and the dose of cell infusion, it remains unclear which factors play decisive roles in CAR-T peak levels in vivo. Endothelial cell dysfunction is consistent with clinical evidence of coagulatory abnormalities and disseminated intravascular coagulation (DIC) in patients with severe encephalic toxicity. Gust et al. [28] demonstrated that severe neurotoxicity was accompanied by DIC, with elevated prothrombin time, activated partial thromboplastin time, and $d$-dimer as early as $2-5$ days after CAR-T cell infusion. Moreover, they found that prolonged thrombocytopenia and a late reduction in fibrinogen to a nadir approximately one to two weeks after CAR-T cell infusion were associated with the development of severe neurotoxicity.

The main difference between CRS and CRES is the IL2 level, which has been associated with severe CRES [22, $25,48,68]$, but not severe CRS. IL-2 stimulates the proliferation and maturation of intracranial cells that are derived from mononuclear macrophages, such as oligodendrocytes and astrocytes [57]. However, whether and to what extent this effect contributes to the development of CRES remains unclear. This discrepancy provides scientists a window to probe into the unique mechanisms underlying the pathogenesis of CRES.

\section{Early prediction}

With plenty of candidates to choose from, the serum levels of IL- 6 and IFN- $\gamma$ in the first $24 \mathrm{~h}$ after CAR-T cell infusion in B-ALL patients have been reported as robust biomarkers of severe CRS and CRES by scientists from the Fred Hutchinson Cancer Research Center (FHCRC) [26, 68]. In NHL patients, high serum levels of IL-8, IL-10, and IL-15, as well as low levels of transforming growth factor (TGF) $-\beta$ could also predict the development of severe CRS and neurotoxicity [68]. Because IL-8, IL-10, IL-15, and TGF- $\beta$ concentrations were not assessed in the BALL cohort of the study NCT01865617, it remains unknown whether these cytokines predict toxicity in BALL. Identification of biomarkers on day one after CAR-T cell infusion provides an opportunity to test whether early intervention strategies in high-risk patients may mitigate or prevent severe toxicity and whether early prevention of severe toxicity will impact the efficacy of CAR-T cells. A combination of robust predictive biomarkers at an early stage needs to be integrated into a precise and sensitive risk grading system to guide therapy decisions.

\section{Biomarkers predicting efficacy}

Factors that can affect the efficacy of CAR-T cell therapy vary, and include the patient and disease characteristics, CAR-T cell culture-related procedures, and lymphodepletion prior to $\mathrm{T}$ cell infusion.

\section{Patient evaluation}

Individual traditional factors, such as patient age, prior therapy, peripheral tumor burden, p53 status $[18,21$, $25]$, the presence of chromosome $17 \mathrm{p}$ deletions, and immunoglobulin heavy chain variable region gene IGH variable (IGHV) mutation status [40], are not associated with response to CAR-T cell therapy. Assessment of the expression of programmed death-1 (PD-1), lymphocyte activation gene-3 (LAG-3), and T cell immunoglobulin-3 (TIM-3) on tumor cells and their receptors on immune cells by immunohistochemical analysis, revealed that high expression of these molecules is associated with a lower response to CTL019 therapy [52]. However, a following study by the same scientists showed contradicting results, with no differences in response based on tumor expression of CD19 or immune checkpointrelated proteins [18]. Therefore, we believe that patients should not be excluded from CAR-T therapy based on age, prior lines of therapy, tumor burden, presence of mutations or expression levels of immune checkpoint molecules, though these characteristics should be taken into account for patient enrollment in clinical trials.

\section{CAR -T cell cultivation}

Several studies, including a phase 2 clinical trial of ZUMA-1, suggested that higher CAR-T- cell levels in the blood are associated with response [25, 44]. Similar results were found in the NCT00924326 study [19] conducted by the National Cancer Institute (NCI) and NCT01029366 conducted by UPenn [40]. Fraietta et al. [21] performed genomic, phenotypic, and functional analyses in 41 CLL patients who were enrolled in clinical trials assessing the use of CTL019 therapy. They identified that $\mathrm{CD}^{+} \mathrm{CD} 45 \mathrm{RO}^{-} \mathrm{CD} 27^{+} \mathrm{T}$ cells in leukapheresis samples were resting, long-lived, memory lymphocytes, which could rapidly expand and acquire effector functions after antigen re-exposure. They also reported that the frequency of these cells was associated with the 
response rate. They also indicated that the population of $\mathrm{CD} 27^{+} \mathrm{PD}-1^{-} \mathrm{CD} 8^{+} \mathrm{CAR}-\mathrm{T}$ cells expressing high levels of the IL- 6 receptor might be associated with response and tumor control. Rossi et al. showed that the pre-infusion of polyfunctional CAR-T cells was significantly associated with clinical response to CAR-T cell therapy [69]. These findings underscore the potential of using biomarkers predicting response in guiding the patient selection and optimizing CAR-T-cell production prior to infusion.

\section{Lymphodepletion}

A recent meta-analysis [70] using data from 320 patients from 14 studies to estimate the response to autologous CD19 CAR-T cell therapy suggested that patients who received lymphodepletion with cyclophosphamide (Cy) or fludarabine (Flu) showed a better response $(77 \%$; $95 \% \mathrm{CI}$, 67-83\%; $P=0.001)$ compared with non-lymphodepleted patients $(66 \%$; $95 \%$ CI, 41-83\%). Furthermore, they found that the administration of higher doses of lymphodepleting agents was associated with higher MCP-1 and IL-7 concentrations after T cell infusion [49], and high MCP-1 and IL-7 levels have been associated with good prognosis. Therefore, whether the extent of lymphodepletion can affect the efficacy of CAR T-cell therapies requires further investigation. Additionally, the optimal duration of lymphodepletion, as well as its combination with other chemotherapies or radiotherapy remain to be established.

\section{Prognostic biomarkers of relapse}

Relapse can occur in up to $50 \%$ of B-ALL patients within 12 months after CAR-T cell infusion. The interaction between the antigen receptor and target plays a decisive role in the efficacy of CAR-T cell therapy; hence, loss or dysfunction of either one may contribute to disease relapse [27, 71]. Early relapse has been reported in antigen-positive patients, while late relapse is typically associated with antigen loss [50]. Antigen-positive relapse usually occurs within the first few months after successful induction of remission and is often associated with limited CAR-T cell persistence and transient B cell aplasia, suggesting a loss of active CAR-T cell-mediated surveillance [23]. Target antigen loss is a well-demonstrated mechanism of disease relapse following successful remission in patients treated with CAR-T cells, yet the mechanisms underlying antigen loss need further investigation.

Scientists from the Seattle Children's Research Institute confirmed that the expansion of CAR-T cells is less robust in patients with short $\mathrm{B}$ cell aplasia (BCA) than in patients with long BCA [47]. They found that the primary driver of CAR-T cell expansion and that minimizes the risk of $\mathrm{CD} 19^{+}$relapse was the cumulative burden of CD19-expressing cells, as assessed in the bone marrow prior to lymphodepleting chemotherapy. In contrast to other reports, neither the cell dose [27] nor leukemia burden $[43,72]$ alone was a predictor of the magnitude or duration of CD19 CAR-T engraftment in this trial. The authors also suggested that a high antigen burden does not induce exhaustion of CAR T-cellsthe therapeutic cells, and that the elimination of the target cells promotes the transition of the effector cells into functional memory CAR-T cells. As with CAR T cell persistency, there are important differences between the $\mathrm{T}$ cell products. For instance, $\mathrm{T}$ cells with 4-1BB co-stimulatory CARs tend to persist longer than $\mathrm{T}$ cells with $\mathrm{CD} 28$ co-stimulatory CARs. 4-1BB-based CAR-T cells persisted in the blood for a median duration of 168 days (range 20-617 days) in patients B cell aplasia who remained in remission, while CD28-based CAR-T cell persistence has been the median duration of 30 days, and these cells are rarely detected beyond 3 months [17]. Phenotypic and functional attributes of CAR-T cells are associated with duration. Finney et al. [47] analyzed the phenotype and function of subtypes in relation to the remission duration. In patients with longterm remission, the percentage of $\mathrm{CD}^{+} \mathrm{CAR}-\mathrm{T}$ cells secreting TNF- $\alpha$ was higher, while the percentage of $\mathrm{CD} 8^{+}$ CAR-T cells expressing TIM-3 was significantly lower. Except for the phenotypes with prognostic value, the frequency of $\mathrm{CD}^{+} \mathrm{CAR}-\mathrm{T}$ cells secreting IFN- $\gamma$ or IL-2, as well as the frequency of $\mathrm{CD}^{+} \mathrm{CAR}$ T-cells expressing PD-1 or LAG-3 did not show significant differences.

The characteristics of the primary disease also have prognostic value. Disease burden detected by the cytological assessment of bone marrow or MRD can predict, to some extent, remission duration. Disease histology can also predict relapse. In a CTL019 lymphoma cohort, the PFS of patients with follicular lymphoma was found to be longer compared to patients with DLBCL [52]. A lower pre-lymphodepletion LDH level (hazard ratio (HR), 1.39; 95\% CI, $1.12-1.74$ per $100 \mathrm{U} / \mathrm{L}$ increment, $P=0.003)$ or a higher pre-lymphodepletion platelet count (HR, 0.65 ; $95 \% \mathrm{CI}, 0.47-0.88$ per $50,000 / \mu \mathrm{L}$ increment increase, $P=0.006$ ) independently associated with better event-free survival (EFS) [50].

Upon interaction between CAR-T cells and tumor cells, complex downstream signaling cascades promote immune cell-mediated cancer cell killing. In the acute setting, biomarkers, such as elevation of cytokines and elimination of certain tumor cell clones, can help identify patients with long-term survival. NHL patients with higher baseline MCP-1 levels or peak IL-7 levels after T cell infusion have a lower incidence of disease progression [49]. Complete remission (CR) after $\mathrm{T}$ cell infusion indicates a longer PFS regardless of the disease type [49, 73] and institution where the study has been conducted $[18,44]$. Among CR patients, high-throughput sequencing (HTS) before and after CAR T-cell infusion revealed that the elimination of leukemic cell clones was 
associated with improved EFS (median event-free survival of 8.4 versus 3.6 months, $P=0.036$ ) [50].

In patients determined as high-risk of relapse after CAR- $T$ therapy, consolidation therapy after infusion will be imperative to prolong survival. A study conducted in our institute highlighted that CAR-T therapy following allogeneic hematopoietic stem cell transplantation (alloHSCT) was a safe and effective therapeutic strategy for relapsed/refractory B-ALL patients, and might prolong EFS and RFS, especially in patients with high preinfusion tumor burden [72]. Hence, the identification of biomarkers is crucial for early identification, accurate intervention, and individualized management.

\section{Challenges and future perspectives}

A major challenge in the development of cancer immunotherapy biomarkers will be the integration of comprehensive bioinformatics data to traditional clinical symptoms, as well as the delineation of the intrinsic mechanisms linking biomarkers to clinical outcomes or phenotypes. Besides biomarkers predicting toxicity, efficacy, and patient survival, extensive efforts to identify novel antigen targets have been made over the last decade, yet with limited breakthrough. The challenge of antigen-receptor design extends to the solid tumor field looking for unique tumor-specific surface antigens and the elucidation of the role of the immunosuppressive tumor microenvironment (TME) [74-76]. Combination therapies provide encouraging clinical benefits in patients with hematologic and solid malignancies. $\mathrm{T}$ cells contain two complete and independent CARs, have overcome, to some extent, the impact of antigen escape in lymphoma [77] and myeloma [78] patients. Novel high-throughput technologies, such as single-cell RNAsequence, for biomarker identification [69], combination therapy with PD-1 inhibitors, and the utilization of universal $\mathrm{T}$ cells may offer virtually unlimited potential for cancer immunotherapy and maximize clinical benefit in cancer patients [79].

\section{Conclusion}

Biomarkers play an important role in personalized management in terms of toxicity, efficacy prediction, and relapse assessment. The discovery of new biomarkers and validation of existing ones should be of high importance so that they can be incorporated into routine clinical practice. The combination of modified CAR-T products and individualized personalized management is imperative to maximize the clinical benefits of CAR-T cell therapy and expand the availability of this promising therapy in a broader range of patients.

\section{Abbreviations}

APTT: Activated partial thromboplastin time; HLH/MAS: Hemophagocytic lymph-histiocytosis/macrophage-activation syndrome; Ang2: Angiopoietin-2;
HTS: High-throughput sequencing; axi-cel: Axicabtagene ciloleucel; IFN: Interferon; B-ALL: B-cell acute lymphocytic leukemia; IGHV: IGH variable: BBB: Blood-brain barrier; IL: Interleukin; BCA: B cell aplasia; IP: Interferon Y induced protein; BCMA: B-cell maturation antigen; LDH: Lactate dehydrogenase; CAR: Chimeric antigen receptor; MCP: Monocyte chemoattractant protein; CLL: Chronic lymphocytic leukemia; MHC: Major histocompatibility complex; CR: Complete remission; MM: Multiple myeloma; CRES: CAR-related encephalopathy syndrome; MRD: Minimal residual disease; CRP: C-reactive protein; MSKCC: Memorial Sloan Kettering Cancer Center;

CRS: Cytokine release syndrome; NCl: National Cancer Institute; CSF: Cerebrospinal fluid; non-NHL: Non- Hodgkin's lymphoma; CTCAE: Common Terminology Criteria for Adverse Events; PFS: Progress-free survival; CTL019: Tisagenlecleucel; PT: Prolonged prothrombin time; Cy: Cyclophosphamide; R/R: Relapse/refractory; DIC: Disseminated intravascular coagulation; sCRES: Severe CRES; DLBCL: Diffuse large B-cell lymphomas; CRS: Severe CRS; EFS: Event-free survival; TGF: Transforming growth factor; FDA: Food and Drug Administration; TLS: Tumor lysis syndrome; FHCRC: Fred Hutchinson Cancer Research Center; TME: Tumor microenvironment; FLT3: Fms-like tyrosine kinase; TNF: Tumor necrosis factor; Flu: Fludarabine; UPenn: University of Pennsylvania; G-CSF: Granulocyte colony-stimulating Factor; vwF: Von Willebrand factor

\section{Acknowledgements}

None.

\section{Authors' contributions}

Heng Mei designed the study. Mengyi Du drafted the manuscript, figure and tables. Parameswaran Hari edited the manuscript. All authors read and approved the final manuscript.

\section{Funding}

This research was supported by the grants from the National Key R\&D Program of China (Grant No 2019YFC1316203); National Natural Science Foundation of China (Grant No 81873434).

\section{Availability of data and materials}

The material supporting the conclusion of this review has been included within the article.

Ethics approval and consent to participate

This is not applicable for this review.

\section{Consent for publication}

This is not applicable for this review.

\section{Competing interests}

The authors declare that they have no competing interests.

\section{Author details}

${ }^{1}$ Institute of Hematology, Union Hospital, Tongji Medical College, Huazhong University of Science and Technology, Wuhan 430022, China. ${ }^{2}$ Division of Hematology/Oncology, Medical College of Wisconsin (MCW), Milwaukee, WI, USA.

Received: 29 January 2020 Accepted: 31 March 2020

Published online: 11 May 2020

\section{References}

1. Hui K, Grosveld F, Festenstein H. Rejection of transplantable AKR leukaemia cells following MHC DNA-mediated cell transformation. Nature. 1984; 311(5988):750-2.

2. Lanitis E, Poussin M, Klattenhoff AW, Song D, Sandaltzopoulos R, June CH, et al. Chimeric antigen receptor $T$ cells with dissociated signaling domains exhibit focused antitumor activity with reduced potential for toxicity in vivo. Cancer Immunol Res. 2013;1(1):43-53.

3. Kong W, Lacey SF, Melenhorst JJ, Fraietta JA. Biomarkers in chimeric antigen receptor T-cell therapy. Biomark Med. 2018;12(5):415-8.

4. Chmielewski M, Hombach AA, Abken H. Of CARs and TRUCKs: chimeric antigen receptor (CAR) T cells engineered with an inducible cytokine to modulate the tumor stroma. Immunol Rev. 2014;257(1):83-90. 
5. Ceppi F, Gardner RA. Chimeric antigen receptor T cells for B-cell acute lymphoblastic leukemia. Cancer J. 2019;25(3):191-8.

6. Frey NV. Chimeric antigen receptor T cells for acute lymphoblastic leukemia. Am J Hematol. 2019;Suppl 94(1):S24-7.

7. Wang J, Hu Y, Huang $\mathrm{H}$. Current development of chimeric antigen receptor T-cell therapy. Stem Cell Investig. 2018;5:44.

8. Drokow EK, Ahmed H, Amponsem-Boateng C, Akpabla GS, Song J, Shi M, et al. Survival outcomes and efficacy of autologous CD19 chimeric antigen receptor-T cell therapy in the patient with diagnosed hematological malignancies: a systematic review and meta-analysis. Ther Clin Risk Manag. 2019;15:637-46

9. Brudno JN, Maric I, Hartman SD, Rose JJ, Wang M, Lam N, et al. T cells genetically modified to express an anti-B-cell maturation antigen chimeric antigen receptor cause remissions of poor-prognosis relapsed multiple myeloma. J Clin Oncol. 2018;36(22):2267-80.

10. June C, Sadelain M. Chimeric antigen receptor therapy. N Engl J Med. 2018; 379(1):64-73.

11. Mikkilineni L, Kochenderfer JN. Chimeric antigen receptor T-cell therapies for multiple myeloma. Blood. 2017;130(24):2594-602.

12. Pan J, Niu Q, Deng B, Liu S, Wu T, Gao Z, et al. CD22 CAR T-cell therapy in refractory or relapsed B acute lymphoblastic leukemia. Leukemia. 2019; 33(12):2854-66.

13. Cummins KD, Gill S. Will CAR T cell therapy have a role in AML? Promises and pitfalls. Semin Hematol. 2019;56(2):155-63.

14. Hofmann S, Schubert ML, Wang L, He B, Neuber B, Dreger $P$, et al. Chimeric antigen receptor (CAR) T cell therapy in acute myeloid leukemia (AML). Clin Med. 2019:8(2):200.

15. Jurgens B, Clarke NS. Evolution of CAR T-cell immunotherapy in terms of patenting activity. Nat Biotechnol. 2019;37(4):370-5.

16. Wang Z, Han W. Biomarkers of cytokine release syndrome and neurotoxicity related to CAR-T cell therapy. Biomark Res. 2018;6(22):4

17. Shah NN, Fry T. Mechanisms of resistance to CAR T cell therapy. Nat Rev Clin Oncol. 2019;16(6):372-85.

18. Schuster SJ, Bishop MR, Tam CS, Waller EK, Borchmann P, McGuirk JP, et al. Tisagenlecleucel in adult relapsed or refractory diffuse large B-cell lymphoma. N Engl J Med. 2019;380(1):45-56.

19. Kochenderfer JN, Somerville R, Lu T, Shi V, Bot A, Rossi J, et al. Lymphoma remissions caused by anti-CD19 chimeric antigen receptor $T$ cells are associated with high serum Interleukin-15 levels. J Clin Oncol. 2017;35(16): 1803-13.

20. Turtle CJ, Hanafi L, Berger C, Gooley TA, Cherian S, Hudecek M, et al. CD19 CAR-T cells of defined CD4+:CD8+ composition in adult B cell ALL patients. J Clin Invest. 2016;126(6):2123-38.

21. Fraietta JA, Lacey SF, Orlando EJ, Pruteanu-Malinici I, Gohil M, Lundh S, et al. Determinants of response and resistance to CD19 chimeric antigen receptor (CAR) T cell therapy of chronic lymphocytic leukemia. Nat Med. 2018;24(5):563.

22. Santomasso B, Park J, Salloum D, Riviere I, Flynn J, Mead E, et al. Clinical and biological correlates of neurotoxicity associated with CAR T-cell therapy in patients with B-cell acute lymphoblastic leukemia. Cancer Discov. 2018;8(8): 958-71

23. Maude SL, Frey N, Shaw PA, Aplenc R, Barrett DM, Bunin NJ, et al. Chimeric antigen receptor $T$ cells for sustained remissions in leukemia. N Engl J Med. 2014;371(16):1507-17.

24. Teachey DT, Lacey SF, Shaw PA, Melenhorst JJ, Maude SL, Frey N, et al. Identification of predictive biomarkers for cytokine release syndrome after chimeric antigen receptor T-cell therapy for acute lymphoblastic leukemia. Cancer Discov. 2016;6(6):664-79.

25. Neelapu SS, Locke FL, Bartlett NL, Lekakis LJ, Miklos DB, Jacobson CA, et al. Axicabtagene Ciloleucel CAR T-cell therapy in refractory large B-cell lymphoma. New Engl J Med. 2017;377(26):2531-44.

26. Hay KA, Hanafi L, Li D, Gust J, Liles WC, Wurfel MM, et al. Kinetics and biomarkers of severe cytokine release syndrome after CD19 chimeric antigen receptor-modified T-cell therapy. Blood. 2017;130(21):2295-306.

27. Gardner RA, Finney O, Annesley C, Brakke H, Summers C, Leger K, et al. Intent-to-treat leukemia remission by CD19 CAR T cells of defined formulation and dose in children and young adults. Blood. 2017;129(25): 3322-31.

28. Gust J, Hay KA, Hanafi LA, Li D, Myerson D, Gonzalez-Cuyar L, et al. Endothelial activation and blood-brain barrier disruption in neurotoxicity after adoptive immunotherapy with CD19 CAR-T cells. Cancer Discov. 2017; 7(12):1404-19.
29. Neelapu SS, Tummala S, Kebriaei P, Wierda W, Gutierrez C, Locke F, et al. Chimeric antigen receptor T-cell therapy - assessment and management of toxicities. Nat Rev Clin Oncol. 2018;15(1):47-62.

30. Park JH, Romero FA, Taur Y, Sadelain M, Brentjens RJ, Hohl TM, et al. Cytokine release syndrome grade as a predictive marker for infections in patients with relapsed or refractory B-cell acute lymphoblastic leukemia treated with chimeric antigen receptor T cells. Clin Infect Dis. 2018;67(4):533-40.

31. Thompson JA, Schneider BJ, Brahmer J, Andrews S, Armand P, Bhatia S, et al. Management of Immunotherapy-Related Toxicities, version 1.2019. J Natl Compr Cancer Netw. 2019;17(3):255-89.

32. Porter D, Frey N, Wood P, Weng Y, Grupp S. Grading of cytokine release syndrome associated with the CAR T cell therapy tisagenlecleucel. J Hematol Oncol. 2018;11(1):35.

33. Liu D, Zhao J. Cytokine release syndrome: grading, modeling, and new therapy. J Hematol Oncol. 2018;11(1):121.

34. Lee DW, Santomasso BD, Locke FL, Ghobadi A, Turtle CJ, Brudno JN, et al. ASTCT consensus grading for cytokine release syndrome and neurologic toxicity associated with immune effector cells. Biol Blood Marrow Transplant. 2019;25(4):625-38.

35. Tisoncik JR, Korth MJ, Simmons CP, Farrar J, Martin TR, Katze MG. Into the eye of the cytokine storm. Microbiol Mol Biol Rev. 2012;76(1):16-32.

36. Oved JH, Barrett DM, Teachey DT. Cellular therapy: immune-related complications. Immunol Rev. 2019;290(1):114-26.

37. Giavridis T, van der Stegen S, Eyquem J, Hamieh M, Piersigilli A, Sadelain M. CAR T cell-induced cytokine release syndrome is mediated by macrophages and abated by IL-1 blockade. Nat Med. 2018;24(6):731-8 2018-06-01.

38. Norelli M, Camisa B, Barbiera G, Falcone L, Purevdorj A, Genua M, et al. Monocyte-derived IL-1 and IL-6 are differentially required for cytokinerelease syndrome and neurotoxicity due to CAR T cells. Nat Med. 2018;24(6): 739-48.

39. Lee DW, Rebecca G, Porter DL, Louis CU, Nabil A, Michael J, et al. Current concepts in the diagnosis and management of cytokine release syndrome. Blood. 2014;124(2):188-95.

40. Porter DL, Hwang WT, Frey NV, Lacey SF, Shaw PA, Loren AW, et al. Chimeric antigen receptor $T$ cells persist and induce sustained remissions in relapsed refractory chronic lymphocytic leukemia. Sci Transl Med. 2015; 7(303):139r-303r.

41. Lee DW, Kochenderfer JN, Stetler-Stevenson M, Cui YK, Delbrook C, Feldman SA, et al. T cells expressing CD19 chimeric antigen receptors for acute lymphoblastic leukaemia in children and young adults: a phase 1 doseescalation trial. Lancet. 2015;385(9967):517-28.

42. Davila ML, Isabelle R, Xiuyan W, Shirley B, Jae P, Kevin C, et al. Efficacy and toxicity management of 19-28z CAR T cell therapy in B cell acute lymphoblastic leukemia. Sci Transl Med. 2014;6(224):224r-5r.

43. Park JH, Riviere I, Gonen M, Wang X, Senechal B, Curran KJ, et al. Long-term follow-up of CD19 CAR therapy in acute lymphoblastic leukemia. N Engl J Med. 2018;378(5):449-59.

44. Locke FL, Ghobadi A, Jacobson CA, Miklos DB, Lekakis L, Oluwole OO, et al. Long-term safety and activity of axicabtagene ciloleucel in refractory large B-cell lymphoma (ZUMA-1): a single-arm, multicentre, phase 1-2 trial. Lancet Oncol. 2019;20(1):31-42.

45. Fitzgerald JC, Weiss SL, Maude SL, Barrett DM, Lacey SF, Melenhorst JJ, et al. Cytokine release syndrome after chimeric antigen receptor $\mathrm{T}$ cell therapy for acute lymphoblastic leukemia. Crit Care Med. 2016;45(2):e124.

46. Karschnia P, Jordan JT, Forst DA, Arrillaga-Romany IC, Batchelor TT, Baehring $J M$, et al. Clinical presentation, management, and biomarkers of neurotoxicity after adoptive immunotherapy with CAR T cells. Blood. 2019; 133(20):2212-21.

47. Finney OC, Brakke HM, Rawlings-Rhea S, Hicks R, Doolittle D, Lopez M, et al. CD19 CAR T cell product and disease attributes predict leukemia remission durability. J Clin Invest. 2019;129(5):2123-32.

48. Gofshteyn JS, Shaw PA, Teachey DT, Grupp SA, Maude S, Banwell B, et al. Neurotoxicity after CTL019 in a pediatric and young adult cohort. Ann Neurol. 2018;84(4):537-46

49. Hirayama AV, Gauthier J, Hay KA, Voutsinas JM, Wu Q, Gooley T, et al. The response to lymphodepletion impacts PFS in patients with aggressive nonHodgkin lymphoma treated with CD19 CAR T cells. Blood. 2019;133(17): 1876-87.

50. Hay KA, Gauthier J, Hirayama AV, Voutsinas JM, Wu Q, Li D, et al. Factors associated with durable EFS in adult B-cell ALL patients achieving MRDnegative CR after CD19 CAR T-cell therapy. Blood. 2019;133(15):1652-63. 
51. Brentjens RJ, Davila ML, Riviere I, Park J, Wang X, Cowell LG, et al. CD19targeted $T$ cells rapidly induce molecular remissions in adults with chemotherapy-refractory acute lymphoblastic leukemia. Sci Transl Med. 2013;177(5):ra38. https://doi.org/10.1126/scitranslmed.3005930.

52. Schuster SJ, Svoboda J, Chong EA, Nasta SD, Mato AR, Anak O, et al. Chimeric antigen receptor T cells in refractory B-cell lymphomas. New Eng J Med. 2017;377(26):2545-54.

53. Gabay C. Interleukin-6 and chronic inflammation. Arthritis Res Ther. 2006; 8(Suppl 2):S3

54. Xing Z. IL-6 is an antiinflammatory cytokine required for controlling local or systemic acute inflammatory responses. J Clin Invest. 1998;101(2):311-20.

55. Yamaoka J, Kabashima K, Kawanishi M, Toda Kl, Miyachi Y. Cytotoxicity of IFN-gamma and TNF-alpha for vascular endothelial cell is mediated by nitric oxide. Biochem Biophys Res Commun. 2002;291(4):780-6.

56. Fiorentino DF, Zlotnik A, Mosmann TR, Howard M, O'Garra A. IL-10 inhibits cytokine production by activated macrophages. J Immunol. 2016;147(11): 3815-22.

57. Liao W, Lin JX, Leonard WJ. Interleukin-2 at the crossroads of effector responses, tolerance, and immunotherapy. Immunity. 2013;38(1):13-25.

58. Lowe KL, Mackall CL, Norry E, Amado R, Jakobsen BK, Binder G. Fludarabine and neurotoxicity in engineered T-cell therapy. Gene Ther. 2018;25(3):176-91.

59. Teachey DT, Rheingold SR, Maude SL, Zugmaier G, Barrett DM, Seif AE, et al. Cytokine release syndrome after blinatumomab treatment related to abnormal macrophage activation and ameliorated with cytokine-directed therapy. Blood. 2013;121(26):5154-7.

60. Bot A, Rossi JM, Jiang $Y$, Navale $L$, Shen $Y$, Sherman $M$, et al. Cyclophosphamide and Fludarabine conditioning chemotherapy induces a key homeostatic cytokine profile in patients prior to CAR T cell therapy. Blood. 2015;126(23):4426.

61. Fiedler U, Reiss $Y$, Scharpfenecker M, Grunow V, Koidl S, Thurston G, et al. Angiopoietin-2 sensitizes endothelial cells to TNF-alpha and has a crucial role in the induction of inflammation. Nat Med. 2006;12(2):235-9.

62. Jiang H, Liu L, Guo T, Wu Y, Ai L, Deng J, et al. Improving the safety of CART cell therapy by controlling CRS-related coagulopathy. Ann Hematol. 2019; 98(7):1721-32

63. Montesinos P, Lorenzo I, Martin G, Sanz J, Perez-Sirvent ML, Martinez D, et al. Tumor lysis syndrome in patients with acute myeloid leukemia: identification of risk factors and development of a predictive model. Haematologica. 2008;93(1):67-74.

64. Cohen LF, Balow JE, Magrath IT, Poplack DG, Ziegler JL. Acute tumor lysis syndrome. A review of 37 patients with Burkitt's lymphoma. Am J Med. 1980;68(4):486-91.

65. Cairo MS, Bishop M. Tumour lysis syndrome: new therapeutic strategies and classification. Br J Haematol. 2004;127(1):3-11.

66. Grupp SA, Kalos M, Barrett D, Aplenc R, Porter DL, Rheingold SR, et al. Chimeric antigen receptor-modified $T$ cells for acute lymphoid leukemia. N Engl J Med. 2013;368(16):1509-18.

67. Hu Y, Sun J, Wu Z, Yu J, Cui Q, Pu C, et al. Predominant cerebral cytokine release syndrome in CD19-directed chimeric antigen receptor-modified $T$ cell therapy. J Hematol Oncol. 2016;9(1):70.

68. Turtle CJ, Hanafi LA, Berger C, Hudecek M, Pender B, Robinson E, et al. Immunotherapy of non-Hodgkin's lymphoma with a defined ratio of CD8+ and CD4+ CD19-specific chimeric antigen receptor-modified T cells. Sci Transl Med. 2016;8(355):116r-355r.

69. Rossi J, Paczkowski P, Shen YW, Morse K, Flynn B, Kaiser A, et al. Preinfusion polyfunctional anti-CD19 chimeric antigen receptor $T$ cells are associated with clinical outcomes in NHL. Blood. 2018;132(8):804-14.

70. Grigor E, Fergusson D, Kekre N, Montroy J, Atkins H, Seftel MD, et al. Risks and benefits of chimeric antigen receptor T-cell (CAR-T) therapy in Cancer: a systematic review and meta-analysis. Transfus Med Rev. 2019;33(2):98-110.

71. Fry TJ, Shah NN, Orentas RJ, Stetler-Stevenson M, Yuan CM, Ramakrishna S, et al. CD22-targeted CAR T cells induce remission in B-ALL that is naive or resistant to CD19-targeted CAR immunotherapy. Nat Med. 2018;24(1):20-8.

72. Jiang H, Li C, Yin P, Guo T, Liu L, Xia L, et al. Anti-CD19 chimeric antigen receptor-modified $T$-cell therapy bridging to allogeneic hematopoietic stem cell transplantation for relapsed/refractory B-cell acute lymphoblastic leukemia: an open-label pragmatic clinical trial. Am J Hematol. 2019;94(10): $1113-22$.

73. Turtle CJ, Hay KA, Hanafi LA, Li D, Cherian S, Chen X, et al. Durable molecular remissions in chronic lymphocytic leukemia treated with CD19- specific chimeric antigen receptor-modified T cells after failure of Ibrutinib. $J$ Clin Oncol. 2017;35(26):3010-20.

74. Wang Z, Wu Z, Liu Y, Han W. New development in CAR-T cell therapy. J Hematol Oncol. 2017;10(1):53.

75. Liu D. Cancer biomarkers for targeted therapy. Biomark Res. 2019;7:25.

76. Liu D. CAR-T "the living drugs", immune checkpoint inhibitors, and precision medicine: a new era of cancer therapy. J Hematol Oncol. 2019;12(1):113.

77. Schultz L, Gardner R. Mechanisms of and approaches to overcoming resistance to immunotherapy. Hematol Am Soc Hematol Educ Program. 2019;2019:1.

78. Lin Q, Zhao J, Song Y, Liu D. Recent updates on CAR T clinical trials for multiple myeloma. Mol Cancer. 2019;18:1541.

79. Liu D, Zhao J, Song Y. Engineering switchable and programmable universal CARs for CAR T therapy. J Hematol Oncol. 2019;12(1):69.

\section{Publisher's Note}

Springer Nature remains neutral with regard to jurisdictional claims in published maps and institutional affiliations.

\section{Ready to submit your research? Choose BMC and benefit from:}

- fast, convenient online submission

- thorough peer review by experienced researchers in your field

- rapid publication on acceptance

- support for research data, including large and complex data types

- gold Open Access which fosters wider collaboration and increased citations

- maximum visibility for your research: over $100 \mathrm{M}$ website views per year

At $\mathrm{BMC}$, research is always in progress.

Learn more biomedcentral.com/submissions 